\title{
$1.53 \mathrm{ST}$
}

\section{Solar Origin Iron Ions in the Earth's Radiation Belts: Multi-dimensional Equilibrium Configuration Modeling with Charge States 1 through 12}

\author{
Spjeldvik, W. N. ${ }^{1}$, Bourdarie, S. ${ }^{2}$, Boscher, D. ${ }^{2}$, Gusev, A. A. ${ }^{3}$, Pugacheva, G. I. ${ }^{3}$ \\ ${ }^{1}$ Physics Department, Weber State University, Ogden, UT 84408-2508, USA, \\ ${ }^{2}$ ONERA-CERT / DESP, 2, avenue Edouard Belin, F-31055 Toulouse, France, \\ ${ }^{3}$ Astrophysics Department (DAS) - Instituto Nacional de Pesquisas Espaciais (INPE) - Av. dos \\ Astronautas, 1758 - Caixa Postal:515 - Cep.:12227-010 São José dos Campos - S.P. - Brasil
}

Energetic IRON (Ferrum) ions are supplied to the Earth's magnetosphere from the processes in the outer parts of the sun (as a component solar wind and as solar energetic particle fluxes). In contrast, the Earth's atmosphere contains very little atomic or ionized iron, except in ooasional meteoric dust. Based on the parameters: geomagnetic L-shell, iron ion magnetic moment, the second adiabatic invariant of these ions, and the ionic charge state number, we have developed a hybrid model for the iron ion distributions in the trapping region. The equilibrium configuration has been computed iteratively, using the technique of stripped derivatives. We have taken care to insure that collisionally induced coupling between the many ionic charge states is numerically treated with algorithms to prevent numerical instability. In this report we show steady state iron ion distributions have been obtained numerically from an assumed outer radiation zone boundary condition at $\mathrm{L}=7$, average values of the radial diffusion coefficients, and standard values for the exospheric neutral densities due to the MSIS- 86 model. We included the twelve lowest ionic charge states for the iron ion for which we have information on the ionic charge exchange cross sections. The computed distributions illustrate the resulting equilibrium structure of energetic iron ions between $10 \mathrm{KeV}$ and $100 \mathrm{MeV}$ ion kinetic energies. The present multi-dimensional results map the magnetic latitudinal extent of the iron ion fluxes. In the central parts of the Earth's radiation belts, computed charge state distributions show the predominance of the lower charge states at the lower energies and the higher charges states in the multi-MeV range. In accordance with solar wind compositional information, we assumed charge state twelve to be the dominant iron ion charge state at the interface between the interplanetary medium and the trapping region, nominally placed at $\mathrm{L}=7$. Like other ion species with multiple available ionic charge states, iron ions, on the average, undergo both a downshift and an upshift in charge state during their diffusive transitory residence in the Earth's inner magnetosphere.

Keywords: radiation belt structure, radial distributions, energetic iron ions, charge states 1 to 12

Corresponding author's e-mail: WSpjeldvik@Weber.edu 